Artículo de investigación

\title{
Reducción de la proteína bruta en la dieta de pollos criollos de engorde en un sistema semi-intensivo
}

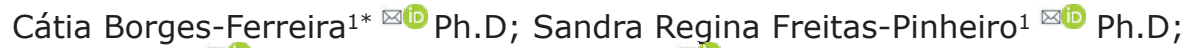

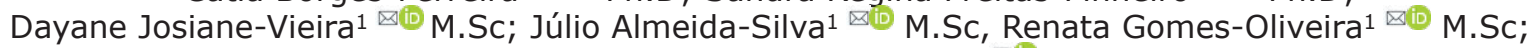 \\ Rúbio Madureira de Souza-Carvalho ${ }^{\circledR(\mathbb{0}}$ M.Sc.
}

${ }^{1}$ Universidade Federal dos Vales do Jequitinhonha e Mucuri (UFVJM), Programa de Pos- Graduación en Zootecnia. Diamantina, MG Brasil. CEP:39100-000.

*Correspondence: sandrafreitaspinheiro@gmail.com

Recibido: Junio 2018; Aceptado: Julio 2019; Publicado: Septiembre 2019.

\section{RESUMEN}

Objetivo. Evaluar la reducción de los niveles de proteína bruta sin alterar la relación de aminoácidos esenciales en pollos de engorde criollos de uno a 42 días de edad. Materiales y métodos. Se realizaron dos ensayos que comprendieron la fase inicial (un a 21 días) y crecimiento ( 22 a 42 días). Se utilizaron 630 pollos de la línea Redbro de un día de edad, distribuidos en un diseño completamente al azar, con cinco tratamientos y seis repeticiones de 21 aves cada una, totalizando 30 unidades experimentales distribuidas en corrales con abrigo. Los niveles reducidos de la proteína bruta para la fase inicial fueron: $21.5 ; 21.0 ; 20.5 ; 20.0$ y $19.5 \%$ y para la fase de crecimiento: $19.0 ; 18.5 ; 18.0 ; 17.5$ y $17.0 \%$. En todos los tratamientos se mantuvo la proporción mínima entre aminoácidos esenciales y lisina. Las características de desempeño evaluadas fueron: ganancia de peso, consumo de ración y conversión alimenticia. Resultados. No hubo diferencia $(p>0.05)$ en la reducción de proteína bruta para ninguna de las variables en todas las fases, excepto para conversión alimenticia $(p<0.05)$ en la fase de crecimiento que mejoró con $18.2 \%$ de proteína bruta. Conclusiones. Los niveles de proteína bruta para pollos criollos de engorde de la línea Redbro, pueden ser reducidos a 19.5 y $18.2 \%$ para la fases inicial y de crecimiento, respectivamente, siempre cuando se mantengan las relaciones adecuadas de aminoácidos con lisina digestible y se atiendan las exigencias de los aminoácidos limitantes.

Palabras clave: aminoácidos esenciales, Label rouge, nutrición, proteína ideal. (Fuente: MeSH, NLM)

\section{ABSTRACT}

Objective. To evaluate the reduction of crude protein levels, without altering the ratio of essential amino acids, of free-range chickens, of one at 42 days old. Materials and methods. Two trials were performed comprising the initial phase (one to 21 days) and growth (22 to 42 days). A total of 630 chicks of the Redbro line of one day old, were distributed in a completely randomized design with five treatments and six replicates of 21 birds each, totaling 30 experimental units consisting of a shelter area and corrals. The levels reduced of crude protein for the initial phase were: $21.5,21.0,20.5,20.0$ and $19.5 \%$ and for the growth phase: $19.0,18.5,18.0,17.5$ and $17.0 \%$. In all the treatments were maintained the minimum ratio between essential amino acids and lysine. The performance characteristics evaluated were: weight gain, feed intake and feed conversion. Results. There was no effect $(p>0.05)$ of the crude protein reduction for any of the variables in all phases, except for feed conversion $(p<0.05)$ that improved with $18.20 \%$ in the growth phase. Conclusions. Crude protein levels for chickens of the Redbro line, can be reduced to $19.5 \%$ and $18.2 \%$ for the initial and growth phases, respectively, provided that the ideal amino acid ratios are maintained with digestible lysine and meet the requirements of limiting amino acids.

Keywords: essential amino acids, ideal protein, Label rouge, nutrition. (Source: MeSH, NLM)

Como citar (Vancouver)

Borges-Ferreira C, Freitas-Pinheiro SR, Josiane-Vieira D; Almeida-Silva J, Gomes-Oliveira R, Souza-Carvalho R. Reducción de la proteína bruta en la dieta de pollos criollos de engorde en un sistema semi-intensivo. Rev MVZ Cordoba. 2019; 24(3):7322-7327. DOI: https://doi.org/10.21897/rmvZ.1824

(c) (1) (C) (los) autor (es), Revista MVZ Córdoba 2019. Este artículo se distribuye bajo los términos de la licencia internacional Creative Commons Attribution 4.0 (https://creativecommons.org/licenses/by-nc-sa/4.0/), que permite a otros distribuir, remezclar, retocar, y crear a partir de su obra de modo no comercial, siempre y cuando den crédito y licencien sus nuevas creaciones bajo las mismas condiciones. 


\section{INTRODUCCIÓN}

La creación de pollos semi-intensivo para la producción de carne con mejores características como el bienestar de los animales, la seguridad alimentaria, la calidad sensorial del producto y la preocupación por el medio ambiente, es una alternativa prometedora del segmento de la avicultura.

Sabiendo que los requisitos para pollos de engorde son aminoácidos esenciales y cantidades suficientes de nitrógeno para la síntesis de proteína bruta Skalan y Plavnik (1) sugieren que las dietas para pollos de engorde deben formularse para proporcionar suficientes aminoácidos para la síntesis de proteínas, ya que el consumo excesivo de aminoácidos puede resultar en una disminución en la eficiencia de la utilización y un aumento en el requerimiento de aminoácidos esenciales. Eso sucede debido a que el exceso de proteína se cataboliza en forma de ácido úrico y este proceso tiene un alto costo energético. Por lo tanto, el uso de niveles menores de proteína, utilizando el concepto de proteína ideal, puede producir un mejor uso del nitrógeno por parte de las aves y disminuir la excreción de este elemento en el medio ambiente.

Cuando Bregendahl et al (2) midieron el efecto de las dietas con bajo contenido de proteína sobre el rendimiento y la composición corporal de los pollos de engorde en la fase inicial, observaron que los pollos alimentados con concentrados que contenían bajo nivel de proteína excretaban menos nitrógeno.

Sin embargo, no hay muchas investigaciones que evalúen los requerimientos de las proteínas para pollos criollos en un sistema de semi-intensivo. Si bien, los requerimientos de proteína de pollos criollos pueden diferir de los pollos de engorda industriales, esta investigación se realizó con el objetivo de evaluar la reducción de la proteína bruta, sin alterar la proporción de aminoácidos esenciales, de los pollos criollos de engorde en un sistema semi-intensivo, en los primeros períodos (1 a 21 días) y crecimiento (22 a 42 días).

\section{MATERIALES Y METODOS}

Localización. El experimento se realizó en junio y agosto de 2012 en el sector avícola del Departamento de Ciencia Animal, Universidade Federal dos Vales de Jequitinhonha e Mucuri, Diamantina, Minas Gerais, Brasil.

Unidades experimentales. El área del cobertizo de cada unidad experimental fue $\left(4 \mathrm{~m}^{2}\right)$ con una altura de $2.5 \mathrm{~m}$, cubierta con tejas de cemento de asbesto, la cerca es compuesta por una malla de alambre y cortinas, piso de cemento y revestimiento de cisco ( $\pm 5 \mathrm{~cm}$ de espesor), con alimentadores manuales y bebedores. El área de pastoreo ( $3 \mathrm{~m}^{2} /$ ave) estaba rodeada por mallas de alambre galvanizado y cubierta por pasto Tifton 85, que caracterizó el sistema de semi-intensivo.

Durante la fase inicial (1 a 21 días), las aves se alojaron en un corral y para mantener la temperatura ideal se utilizaron lámparas infrarrojo de $250 \mathrm{~W}$.

Para la siguiente fase (22 a 42 días), las aves fueron transferidas (a los 22 días) a sus respectivas unidades experimentales y a partir del día 28 tuvieron acceso al área de pastoreo y liberadas en el horario de 8:00 a 18:00 h. Estas aves fueron criadas en las mismas condiciones hasta el día 21 y luego transferidas a sus respectivas unidades experimentales, estas aves recibieron dietas formuladas para cumplir con sus requisitos, de acuerdo con las recomendaciones de Rostagno et al (3).

La temperatura promedio mínima y máxima registrada en el interior de la casa fue de 28.5 y $32.5^{\circ} \mathrm{C}$ para la fase inicial y para la fase de crecimiento fue de 26.5 y $29^{\circ} \mathrm{C}$, respectivamente.

Animales, dietas y diseño experimental. En cada experimento, se distribuyeron 630 pollos criollos machos de la línea de Redbro en un diseño completamente al azar con cinco tratamientos y seis réplicas de 21 aves por unidad experimental.

Para cada fase experimental, se formularon dietas compuestas principalmente de maíz y harina de soya, para ser isoenergéticas y cumplir con todas las exigencias nutricionales de los pollos. Las dietas fueron formuladas siguiendo las tablas nutricionales de Rostagno et al (3) para pollos de desempeño regular, excepto para proteína bruta. Por otro lado, los cálculos descritos por Nagib et al (4), Pinheiro et al (5) y Pinheiro et al (6) se utilizaron respectivamente para atender los requerimientos nutricionales de lisina digestible, fósforo disponible y calcio.

Los niveles decrecientes de proteína bruta dietética (tratamientos) fueron de 21.5, 21.0, 20.5, $20.0 \mathrm{y}$ $19.5 \%$, y $19.0,18.5,18.0,17.5$ y $17.0 \%$ para los períodos inicial (Tabla 1) y en crecimiento (Tabla 2) respectivamente. Los niveles de proteína bruta se obtuvieron ajustando las cantidades de maíz, harina de soya y gluten de maíz. En todos los tratamientos se mantuvo la relación mínima entre aminoácidos esenciales y lisina como propuso Rostagno et al (3), mediante la suplementación de aminoácidos industriales. Durante los primeros 5 días de vida, todos los pollos recibieron un complejo vitamínico diluido en agua. 
Tabla 1. Composición porcentual de las dietas experimentales para pollos de engorde durante el período inicial (1 a 21 días).

\begin{tabular}{|c|c|c|c|c|c|}
\hline \multirow{2}{*}{ Ingrediente } & \multicolumn{5}{|c|}{ Proteína Bruta (\%) } \\
\hline & 21.50 & 21.00 & 20.50 & 20.00 & 19.50 \\
\hline Maíz & 57.951 & 59.732 & 61.033 & 62.563 & 65.252 \\
\hline Harina de soja $45 \%$ & 35.716 & 34.175 & 33.458 & 31.712 & 29.250 \\
\hline Fosfato dicálcico & 1.522 & 1.529 & 1.535 & 1.542 & 1.551 \\
\hline Aceite de soja & 1.571 & 1.247 & 1.123 & 0.749 & 0.203 \\
\hline Harina glúten de maíz 60\% & 1.127 & 1.142 & 0.634 & 0.779 & 1.359 \\
\hline Caliza & 1.221 & 1.225 & 1.225 & 1.230 & 1.237 \\
\hline Sal & 0.494 & 0.494 & 0.494 & 0.494 & 0.494 \\
\hline Premezcla de Vitaminas (1) & 0.100 & 0.100 & 0.100 & 0.100 & 0.100 \\
\hline Premezcla Mineral (2) & 0.050 & 0.050 & 0.050 & 0.050 & 0.050 \\
\hline DL-metionina (99\%) & 0.154 & 0.166 & 0.182 & 0.193 & 0.201 \\
\hline L-lisina $(78.0 \%)$ & 0.004 & 0.050 & 0.077 & 0.128 & 0.198 \\
\hline L-treonina (99\%) & 0.000 & 0.000 & 0.000 & 0.000 & 0.016 \\
\hline Cloruro de colina $(60 \%)$ & 0.040 & 0.040 & 0.040 & 0.040 & 0.040 \\
\hline Antioxidante (3) & 0.050 & 0.050 & 0.050 & 0.050 & 0.050 \\
\hline \multirow[t]{2}{*}{ TOTAL } & 100.00 & 100.00 & 100.00 & 100.00 & 100.00 \\
\hline & \multicolumn{5}{|c|}{ Composición calculada } \\
\hline Proteína bruta (\%) & 21.50 & 21.00 & 20.50 & 20.00 & 19.50 \\
\hline Energía metabolizable (kcal/kg) & 2952 & 2952 & 2952 & 2952 & 2952 \\
\hline Calcio (\%) & 0.950 & 0.950 & 0.950 & 0.950 & 0.950 \\
\hline Fósforo disponible (\%) & 0.394 & 0.394 & 0.394 & 0.394 & 0.394 \\
\hline Sódio (\%) & 0.215 & 0.215 & 0.215 & 0.215 & 0.215 \\
\hline $\begin{array}{l}\text { Metionina + cisteína } \\
\text { digestible }(\%)\end{array}$ & 0.750 & 0.750 & 0.750 & 0.750 & 0.750 \\
\hline Metionina digestible (\%) & 0.449 & 0.455 & 0.462 & 0.468 & 0.473 \\
\hline Lisina digestible (\%) & 1.041 & 1.041 & 1.041 & 1.041 & 1.041 \\
\hline Treonina digestible (\%) & 0.738 & 0.720 & 0.702 & 0.682 & 0.677 \\
\hline Arginina digestible (\%) & 1.350 & 1.308 & 1.280 & 1.234 & 1.174 \\
\hline Triptófano digestible (\%) & 0.239 & 0.231 & 0.226 & 0.217 & 0.206 \\
\hline Valina digestible (\%) & 0.925 & 0.901 & 0.877 & 0.853 & 0.828 \\
\hline
\end{tabular}

(1) por quilograma do produto: Vitamina A - 12 milliones IU, vit. D3 $2.200,000 \mathrm{IU}$, vit. E - $30 \mathrm{~g}$, vit. B1 - $2.2 \mathrm{~g}$, vit. B2 $-6 \mathrm{~g}$, vit. B6 - $3.3 \mathrm{~g}$, vit. B12 - $0.016 \mathrm{mcg}$, Ácido Pantotenico- $13 \mathrm{~g}$, vit. K - 3 a $2.5 \mathrm{~g}$, acido folico - 1 $\mathrm{g}$, antioxidante $-100.000 \mathrm{mg}$ and veiculo qsp $-1.000 \mathrm{~g}$.

(2) por quilograma do produto: Manganeso, $75.000 \mathrm{mg}$, hierro, $50.000 \mathrm{mg}$, zinc, $70.000 \mathrm{mg}$, copper, $8.500 \mathrm{mg}$, cobalt, $200 \mathrm{mg}$, iodine, $1.500 \mathrm{mg}$ and vehicle qsp $1000 \mathrm{~g}$.

(3) Hidroxitolueno butilado.

Desempeño. Los parámetros de desempeño evaluados fueron: ganancia de peso (g/ave), ingesta de ración ( $\mathrm{g} / \mathrm{ave}$ ) y conversión alimenticia ( $\mathrm{g}$ ingesta de ración/g ganancia de peso) corregida por la mortalidad. Las aves se pesaron al principio y al final de cada experimento para determinar la ganancia de peso. Las aves fueron sometidas a 12 horas de ayuno antes de la matanza. Se usó la diferencia entre la dieta ofrecida y las sobras para obtener la ingesta de ración. Para el cálculo de la conversión alimenticia se utilizó la relación entre la ingesta de ración y la ganancia de peso.

Análisis estadístico. El análisis de varianza, incluido el efecto fijo de los niveles de proteína bruta dietética en el modelo, se realizó mediante el software del Sistema de Análisis Estadístico (7).
Tabla 2. Composición porcentual de las dietas experimentales para pollos de engorde durante el período crecimiento (22 a 42 días).

\begin{tabular}{|c|c|c|c|c|c|}
\hline \multirow{2}{*}{ Ingredients } & \multicolumn{5}{|c|}{ Proteína Bruta (\%) } \\
\hline & 19.00 & 18.50 & 18.00 & 17.50 & 17.00 \\
\hline Maíz & 63.375 & 65.172 & 66.989 & 69.118 & 71.328 \\
\hline Harina de soja $45 \%$ & 30.436 & 28.872 & 27.256 & 25.266 & 23.184 \\
\hline Fosfato dicálcico & 1.327 & 1.335 & 1.342 & 1.353 & 1.363 \\
\hline Aceite de soja & 2.620 & 2.294 & 1.972 & 1.596 & 1.208 \\
\hline Caliza & 1.197 & 1.201 & 1.205 & 1.209 & 1.213 \\
\hline Sal & 0.457 & 0.456 & 0.456 & 0.456 & 0.456 \\
\hline Premezcla de Vitaminas (1) & 0.100 & 0.100 & 0.100 & 0.100 & 0.100 \\
\hline Premezcla Mineral (2) & 0.050 & 0.050 & 0.050 & 0.050 & 0.050 \\
\hline DL-metionina (99\%) & 0.208 & 0.221 & 0.234 & 0.250 & 0.268 \\
\hline L-lisina $(78.0 \%)$ & 0.134 & 0.181 & 0.230 & 0.291 & 0.355 \\
\hline L-triptofano (99\%) & 0.000 & 0.000 & 0.000 & 0.000 & 0.012 \\
\hline L-treonina (99\%) & 0.006 & 0.026 & 0.047 & 0.072 & 0.100 \\
\hline L-arginina $(93.1 \%)$ & 0.000 & 0.000 & 0.000 & 0.055 & 0.118 \\
\hline L-valina $(96.5 \%)$ & 0.000 & 0.002 & 0.029 & 0.061 & 0.097 \\
\hline L-isoleucina $(96.5 \%)$ & 0.000 & 0.000 & 0.000 & 0.033 & 0.069 \\
\hline Cloruro de colina (60\%) & 0.040 & 0.040 & 0.040 & 0.040 & 0.040 \\
\hline Antioxidante ${ }^{(3)}$ & 0.050 & 0.050 & 0.050 & 0.050 & 0.050 \\
\hline \multirow[t]{2}{*}{ TOTAL } & 100.0 & 100.0 & 100.0 & 100.0 & 100.0 \\
\hline & \multicolumn{5}{|c|}{ Composición calculada } \\
\hline Proteína bruta (\%) & 19.00 & 18.50 & 18,00 & 17.50 & 17.00 \\
\hline Energía metabolizable (kcal/kg) & 3075 & 3075 & 3075 & 3075 & 3075 \\
\hline Calcio (\%) & 0.881 & 0.881 & 0,881 & 0.881 & 0.881 \\
\hline Fósforo disponible (\%) & 0.351 & 0.351 & 0,351 & 0.351 & 0.351 \\
\hline Sódio (\%) & 0.200 & 0.200 & 0,200 & 0.200 & 0.200 \\
\hline $\begin{array}{l}\text { Metionina + cisteína } \\
\text { digestible }(\%)\end{array}$ & 0.734 & 0.734 & 0,734 & 0.734 & 0.734 \\
\hline Metionina digestible (\%) & 0.466 & 0.473 & 0,479 & 0.487 & 0.497 \\
\hline Lisina digestible (\%) & 1.006 & 1.006 & 1,006 & 1.006 & 1.006 \\
\hline Treonina digestible (\%) & 0.654 & 0.654 & 0,654 & 0.654 & 0.654 \\
\hline Arginina digestible (\%) & 1.180 & 1.136 & 1,091 & 1.087 & 1.087 \\
\hline Triptófano digestible (\%) & 0.208 & 0.200 & 0.191 & 0.181 & 0.181 \\
\hline Valina digestible (\%) & 0.808 & 0.785 & 0.786 & 0.785 & 0.785 \\
\hline \multicolumn{6}{|c|}{$\begin{array}{l}\text { (1) por quilograma do produto: Vitamina A - } 12 \text { milliones IU, vit. D3 - } \\
2.200,000 \mathrm{IU} \text {, vit. E }-30 \mathrm{~g} \text {, vit. B1 }-2.2 \mathrm{~g} \text {, vit. B2 }-6 \mathrm{~g} \text {, vit. B6 }-3.3 \mathrm{~g} \text {, vit. } \\
\mathrm{B} 12-0.016 \mathrm{mcg} \text {, Acido Pantotenico- } 13 \mathrm{~g} \text {, vit. } \mathrm{K}-3 \mathrm{a} 2.5 \mathrm{~g} \text {, acido folico }-1 \\
\mathrm{~g} \text {, antioxidante }-100.000 \mathrm{mg} \text { and veiculo qsp }-1.000 \mathrm{~g} \text {. } \\
\text { (2) por quilograma do produto: Manganeso, } 75.000 \mathrm{mg} \text {, hierro, } 50.000 \mathrm{mg} \text {, } \\
\text { zinc, } 70.000 \mathrm{mg} \text {, copper, } 8.500 \mathrm{mg} \text {, cobalt, } 200 \mathrm{mg} \text {, iodine, } 1.500 \mathrm{mg} \text { and } \\
\text { vehicle qsp } 1000 \mathrm{~g} \text {. } \\
\text { (3) Hidroxitolueno butilado. }\end{array}$} \\
\hline
\end{tabular}

Cuando se detectó un efecto significativo $(p<0.05)$, se realizó un análisis de regresión. Los modelos lineales, linear response plateau (LRP) o cuadráticos ( $\beta 1 \circ \beta 2 \neq 0$, respectivamente; $p<0.05)$ se eligieron en función de los coeficientes de determinación $\left(R^{2}\right)$.

Comité de ética y bioseguridad. El experimento fue aprobado por el Comité de Ética sobre el Uso de Animales (ECUA) número 005/11 de la Universidade Federal dos Vales de Jequitinhonha e Mucuri, Diamantina, Minas Gerais, Brasil. 


\section{RESULTADOS}

Los resultados de desempeño para los períodos inicial y crecimiento se detallan en la tabla 3. En el período inicial de 1 a 21 días de edad, los niveles de proteína bruta no influyeron ( $p>0.05)$ en la ingesta de la ración, la ganancia de peso y la conversión alimenticia de los pollos criollos Redbro.

Tabla 3. Medias y desviación estándar para el consumo de ración (CR), ganancia de peso (GP) y la conversión alimenticia (CA) de los pollos criollos de engorde de la línea Redbro, que recibieron niveles decrecientes de proteína bruta en la dieta durante los períodos iniciales ( 1 a 21 días) y crecimiento (22 a 42 días).

\begin{tabular}{|c|c|c|c|c|c|c|c|}
\hline \multicolumn{8}{|c|}{ Período Inicial (1 a 21 días) } \\
\hline \multirow{2}{*}{ Variables } & \multicolumn{7}{|c|}{ Reducción Proteína (\%) } \\
\hline & 21.5 & 21.0 & 20.5 & 20.0 & 19.5 & p valor & $\begin{array}{l}\text { CV } \\
(\%)\end{array}$ \\
\hline CR (g) & $\begin{array}{c}929 \\
\pm 46.39\end{array}$ & $\begin{array}{c}921 \\
\pm 43.91\end{array}$ & $\begin{array}{c}889 \\
\pm 49.66\end{array}$ & $\begin{array}{c}898 \\
\pm 29.00\end{array}$ & $\begin{array}{c}922 \\
\pm 64.69\end{array}$ & 0.33 & 5.34 \\
\hline GP $(g)$ & $\begin{array}{c}519 \\
\pm 16.83\end{array}$ & $\begin{array}{c}477 \\
\pm 18.19\end{array}$ & $\begin{array}{c}503 \\
\pm 10.87\end{array}$ & $\begin{array}{c}516 \\
\pm 16.75\end{array}$ & $\begin{array}{c}476 \\
\pm 27.91\end{array}$ & 0.31 & 3.81 \\
\hline $\mathrm{CA}(\mathrm{g} / \mathrm{g})$ & $\begin{array}{l}1.789 \\
\pm 0.06\end{array}$ & $\begin{array}{l}1.931 \\
\pm 0.07\end{array}$ & $\begin{array}{l}1.766 \\
\pm 0.08\end{array}$ & $\begin{array}{l}1.740 \\
\pm 0.02\end{array}$ & $\begin{array}{l}1.938 \\
\pm 0.11 \\
\end{array}$ & 0.28 & 4.23 \\
\hline \multicolumn{8}{|c|}{ Período Crecimiento ( 22 a 42 días) } \\
\hline \multirow{2}{*}{ Variables } & \multicolumn{7}{|c|}{ Reducción Proteína (\%) } \\
\hline & 19.0 & 18.5 & 18.0 & 17.5 & 17.0 & p valor & $C V(\%)$ \\
\hline CR $(g)$ & $\begin{array}{l}2.322 \\
\pm 0.04\end{array}$ & $\begin{array}{l}2.336 \\
\pm 0.03\end{array}$ & $\begin{array}{l}2.311 \\
\pm 0.06\end{array}$ & $\begin{array}{l}2.372 \\
\pm 0.03\end{array}$ & $\begin{array}{l}2.354 \\
\pm 0.03\end{array}$ & 0.25 & 1.77 \\
\hline GP $(g)$ & $\begin{array}{l}1.110 \\
\pm 0.04\end{array}$ & $\begin{array}{l}1.121 \\
\pm 0.07\end{array}$ & $\begin{array}{l}1.088 \\
\pm 0.04\end{array}$ & $\begin{array}{l}1.104 \\
\pm 0.02\end{array}$ & $\begin{array}{l}1.056 \\
\pm 0.07\end{array}$ & 0.14 & 4.83 \\
\hline $\mathrm{CA}(\mathrm{g} / \mathrm{g})^{1,2}$ & $\begin{array}{l}2.094 \\
\pm 0.06\end{array}$ & $\begin{array}{l}2.091 \\
\pm 0.14\end{array}$ & $\begin{array}{l}2.126 \\
\pm 0.07\end{array}$ & $\begin{array}{l}2.148 \\
\pm 0.02\end{array}$ & $\begin{array}{l}2.239 \\
\pm 0.18\end{array}$ & 0.02 & 5.23 \\
\hline
\end{tabular}

$\mathrm{CV}=$ coeficiente de variación.

1Modelo linear: $C A=3.4001-0.0699 P B\left(R^{2}=0.82\right)$

${ }^{2}$ Linear Response Plateau model: $C A=2.0928-0.1125(P B-18.206)$ $\left(R^{2}=0.36\right)$.

Para el período de crecimiento (22 a 42 días), la reducción de la proteína bruta no afectó $(p>0.05)$ la ingesta de ración y la ganancia de peso de los pollos.

Para la conversión de la alimenticia (CA) se observó un efecto significativo $(p<0.05)$ y se ajustó por el modelo linear e LRP. Por la estimativa obtenida en el modelo LRP con nivel de $18.206 \%$ de PB se obtuvo la mejor CA de las aves y a partir de este nivel, no hubo cambios.

\section{DISCUSIÓN}

En el presente estudio se evaluó la reducción de la proteína bruta sin alterar la proporción de aminoácidos esenciales de los pollos criollos. El consumo de ración, la ganancia de peso y la conversión alimenticia fueron analizadas para comprender la acción de la proteína bruta en el desempeño de las aves.
Los datos muestran, que, para el período inicial, de 1 a 21 días de edad, que la reducción de proteína no influyó en las características evaluadas.

Estos resultados demostraron que es posible reducir el nivel dietético de la proteína en la fase inicial para $19.5 \%$, si la formulación es basada en aminoácidos esenciales. La ausencia de efecto puede ser justificada por el hecho de que, aunque las raciones tengan diferentes niveles de proteína bruta, se mantiene la relación mínima de lisina con los otros aminoácidos esenciales en todos los niveles estudiados. Este procedimiento puede caracterizar el uso de los aminoácidos esenciales a expensas de la proteína bruta.

De acuerdo con Diambra y Mccartney (8), las aves sometidas a ración con niveles reducidos de proteínas tienden a aumentar la ingesta en un intento de compensar las posibles deficiencias de proteínas o aminoácidos. Este hecho no se observó en este estudio, posiblemente debido a una suplementación adecuada con aminoácidos esenciales. Por lo tanto, no hubo una gran variación en los resultados, lo que justifica que los pollos criollos al igual que otros linajes de aves, requieren los aminoácidos esenciales mínimos y no sólo la proteína bruta.

Resultados similares fueron observados por Rocha et al (9) que al evaluar los niveles de proteína para pollos en la fase 1 a 21 días de edad, no encontraron diferencias significativas para la ganancia de peso y la conversión alimenticia. Sin embargo, observaron un aumento en el consumo de ración a un nivel del $20 \%$, con relación al nivel del $23 \%$. Thon et al (10) evaluaron diferentes niveles de proteína bruta y arginina en la ración de pollos de 1 a 14 días y observaron una mejor ganancia de peso, con el nivel de proteína más alto (22\%) estudiado. Vasconcellos et al (11) observaron una reducción en el rendimiento de los pollos de 14 dias y observaron engorde durante la fase inicial cuando redujeron los niveles de proteína del 23 al $17 \%$, lo que sugiere que la suplementación con aminoácidos esenciales no era suficiente para satisfacer las exigencias de los animales.

Para medir el efecto de la ración con baja proteína en el rendimiento y la composición corporal de los pollos en la fase inicial, Bregendahl et al (2) señalaron que los pollos alimentados con una ración de proteína baja crecieron más lentamente y utilizaron los alimentos de manera menos eficiente; sin embargo, excretan menos nitrógeno al el medio ambiente, habiendo sido reportado este efecto positivo (2).

La reducción de la proteína bruta en el período de crecimiento no influyó en la ganancia de peso ni en la ingesta de ración. Hubo un efecto significativo para la conversión alimenticia con mejor el nivel de proteína bruta del $18.20 \%$. 
Estos resultados demuestran que la formulación de ración basada en el concepto de proteína ideal, con una suplementación adecuada de aminoácidos industriales, es capaz de garantizar el rendimiento de los pollos de engorde de linaje de crecimiento lento en esta etapa.

Se encontraron resultados similares con Costa et al (12) que estudiaron dietas con diferentes niveles de PB (19.5 até $17.5 \%$ ) para pollos de engorde de 22 a 42 días, no se observaron diferencias significativas en la ganancia de peso, lo que sugiere que para esta variable los niveles se pueden reducir a $17.5 \%$.

De la misma manera, Nagata et al (13) encontraron resultados positivos cuando se redujeron los niveles de proteína en las dietas de pollos de engorde en la fase de terminación, en donde las dietas fueron suplementadas con aminoácidos y la enzima fitasa. Sin embargo, Leandro et al (14) evaluando diferentes planos nutricionales y niveles de proteína bruta $(18.50,17.77,16.95$ y $16.68 \%)$ y energía metabolizable, mostraron que los pollos tenían mejor ganancia de peso y conversión alimenticia, hasta los 39 días de edad, con niveles elevados de proteína.

Según Silva et al (15), el hecho de que las aves no alcancen un rendimiento óptimo, empleando dietas con niveles bajos de PB se ha atribuido a factores como: niveles bajos de potasio (debido a la disminución de la cantidad de harina de soya); cambios en el equilibrio iónico; falta de nitrógeno suficiente para a síntesis de aminoácidos no esenciales. Además desequilibrio entre ciertos aminoácidos tales como arginina y lisina o aminoácidos de cadena ramificada; posibles niveles tóxicos de ciertos aminoácidos y la proporción inadecuada de triptófano y otros aminoácidos neutros (isoleucina, valina, leucina, fenilalanina y residuos de tirosina), que pueden inhibir la ingesta de ración.
Mendoza et al (16) encontraron que el costo de la ración fue más alto para aquellas que eran formuladas con el concepto de proteína ideal; sin embargo, debido a que han proporcionado un mayor peso a los pollos de engorde, tuvieron costos por ganancia más bajos, siendo $3.5 \%$ mejores.

Es común obtener información de la literatura en la que los autores informen una posible reducción de los costos al evaluar las dietas con el concepto de proteína ideal. Además, los niveles más bajos de proteína bruta y la suplementación con aminoácidos esenciales pueden tener efectos beneficiosos con la mayor retención de nitrógeno, con reducción de la contaminación ambiental (2).

Los niveles de proteína para los pollos criollos de la línea Redbro se pueden reducir al $19.5 \%$ para el período inicial ( 1 a 21 días) y al $18.2 \%$ para el período de crecimiento (22 a 42 días) sin afectar el rendimiento zootécnico, siempre que la proporción ideal de aminoácidos-lisina digestible sea alcanzada y se mantengan las exigencias de los aminoácidos limitantes.

\section{Conflicto de intereses.}

Los autores del presente estudio declaramos que no existe conflicto de intereses con la publicación de este manuscrito.

\section{Agradecimientos}

Agradecemos a la Fundación de Investigación del Estado de Minas Gerais (FAPEMIG) por el apoyo financiero y a Ajinomoto do Brasil Industria y Comercio de Alimentos Ltda. por la donación de los aminoácidos y el reconocimiento al profesor Aldrin Vieira Pires (en memoria) por su contribución en este estudio.

\section{REFERENCIAS}

1. Skalan D, Plavnik I. Interactions between dietary crude protein and essencial amino acid intake on performance in broilers. $\mathrm{Br}$ Poul Sci 2002; 43(3):442-449. https://doi. org/10.1080/00071660120103710

2. Bregendahl K, Sell JL, Zimmerman DR. Effect of low-protein diets on growth performance and body composition of broiler chicks. Poul Sci 2002; 81(10):1156-1167. https://doi. org/10.1093/ps/81.8.1156
3. Rostagno HS, et al. Tabelas brasileiras para aves e suínos: composição de alimentos e exigências nutricionais. 3. ed. - Viçosa, Minas Gerais: Universidade Federal de Viçosa, departamento de Zootecnia; 2011. https:// www.agencia.cnptia.embrapa.br/Repositorio/ Tabelas+brasileiras+-+Rostagno 000gy1tqv m602wx7ha0b6gs0xfzo6pk5.pdf

4. Nagib NDC, Sakomura NK, Siqueira JC, Dourado LRB, Fernandes JBK, Malheiros EB. Exigências de lisina digestível para aves de corte da linhagem ISA Label criadas em semiconfinamento. Arq Bras Med Vet Zootec 2009; 61(5):1128-1138. https://doi. org/10.1590/S0102-09352009000500016 
5. Pinheiro SRF, Sakomura NK, Nagib NDC, Dourado LRB, Fernandes, JBK, Thomaz, MC. Níveis nutricionais de fósforo disponível para aves de corte ISA Label criadas em semiconfinamento. R Bras Zootec 2011a; 40(2):361-369. https://doi.org/10.1590/ $\underline{\mathrm{S} 1516-35982011000200018}$

6. Pinheiro SRF, Sakomura NK, Siqueira JC, Marcato SM, Dourado LRB, Fernandes JBK et al. Níveis nutricionais de cálcio para aves de corte ISA Label criadas sob semiconfinamento. Arq Bras Med Vet Zootec 2011b; 63(1):231238. https://doi.org/10.1590/S0102$\underline{09352011000100033}$

7. SAS Institute: statistical analysis system [Software]. Version. 8.02. edition. SAS Institute Inc., Cary, NC 2001. https://www. sas.com/es co/software/stat.html

8. Diambra $\mathrm{OH}$, Mccartney MG. The effect of low protein finisher diets on broiler males performance and abdominal fat. Poul Sci 1995; 64(10):2013-2015. https://doi. org/10.3382/ps.0642013

9. Rocha PT, Stringhini JH, Andrade MA, Leandro NSM, Andrade ML, Café MB. Desempenho de frangos de corte alimentados com rações pré-iniciais contendo diferentes níveis de proteína bruta e energia metabolizável. R Bras Zootec 2003; 32(1):162-170. https://doi. org/10.1590/S1516-35982003000100021

10. Thon MS, Stringhini JH, Filho RMJ, Andrade MA, Café MB, Leandro NSM. Níveis de proteína e de arginina digestível na ração pré-inicial de frangos de corte. R Bras Zootec 2010; 39(5):1105-1111. https://doi.org/10.1590/ $\underline{\mathrm{S} 1516-35982010000500022}$

11. Vasconcellos CHF, Fontes, DO, Corrêa GSS, Vidal TZB, Silva MA, Machado ALC et al. Efeitos da redução da proteína dietética sobre o desempenho e as características de carcaça de frangos de corte de 1 a 21 dias de idade. R Bras Zootec 2012; 41(3):662-667. https://doi.org/10.1590/ $\underline{\mathrm{S} 1516-35982012000300028}$
12. Costa FGP, Rostagno HS, Albino LFT, Gomes PC, Toledo RS, Junior JGV. Níveis dietéticos de proteína bruta para frangos de corte de 1 a 21 e 22 a 42 dias de idade. R Bras Zootec 2001; 30(5): 1498-1505. https://doi.org/10.1590/ S1516-35982001000600017

13. Nagata $A K$, Rodrigues $P B$, Rodrigues KF, Freitas RTF, Albino LFT, Fialho ET. Uso do conceito de proteína ideal em rações com diferentes níveis energéticos, suplementadas com fitase para frangos de corte no período de 1 a 21 dias de idade. Ciên Agrotec 2009; 33(2):599-605. https://doi.org/10.1590/S1413$\underline{70542009000200037}$

14. Leandro NSM, Café MB, Stringhini RMF, Moura KA, Júnior RPS. Plano nutricional com diferentes níveis de proteína bruta e energia metabolizável na ração, para frangos de corte. R Bras Zootec 2003; 2(3):620-631. https://doi.org/10.1590/ $\underline{\mathrm{S} 1516-35982003000300014}$

15. Silva $Y L$, Rodrigues PB, Freitas RTF, Bertechini $A B$, Fialho ET, Fassani EJ et al. Redução de proteína e fósforo em rações com fitase para frangos de corte no período de 1 a 21 dias de idade: desempenho e teores de minerais na cama. R Bras Zootec 2006; 35(5):840-848. https://doi.org/10.1590/S151635982006000300029

16. Mendoza MOB, Costa PTC, Katzer LH, Beetti AC, Santi ZB, Welter JN. Desempenho de frangos de corte, sexados, submetidos a dietas formuladas pelos conceitos de proteína bruta versus proteína ideal. Cien Rural 2001; 31(1):111-115. https://doi.org/10.1590/ $\underline{\mathrm{S} 0103-84782001000100018}$ 